\title{
Long Chain Fatty Acid
}

National Cancer Institute

\section{Source}

National Cancer Institute. Long Chain Fatty Acid. NCI Thesaurus. Code C68388.

A class of dietary fatty acids that have an alkyl chain length from 13 up to 21 carbon atoms. 